\title{
Estudio microanalítico de los cursos de acción cooperativos y competitivos en niños y adolescentes
}

\author{
M. J Jesús Fuentes y Pablo Fernández \\ Universidad de Málaga
}

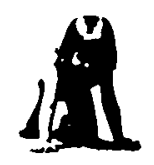

Resumen

En este estudio se analizan los efectos de variables afectivas como la empatía, la ansiedad y la amistad en las interacciones cooperativas o competitivas de los sujetos desde un punto de vista evolutivo. Los objetivos fueron: analizar la influencia de la empatía y la ansiedad en las interacciones cooperativas, y explorar las diferencias evolutivas en los cursos de acción cooperativos de los sujetos cuando la relación que existía entre ellos era de mutua amistad o de mutuo rechazo. Las interacciones se anali. zaron mediante «Análisis Secuencial de Retardos» (Sackett, 1980) para conocer los procesos interactivos. Se obtuvieron patrones conductuales diferenciados en los niños y en los adolescentes en función de la empatía y las relaciones de amistad existentes entre los sujetos.

Palabras clave: Empatía; Amistad; Cooperación; Competición; Niños; Adolescentes.

\section{Micro-analytical study of cooperative and competitive courses of action in children and adolescents}

\begin{abstract}
In this study the effects of affective variables such as empathy, anxiety, and friendship in cooperative or competitive interactions are analysed from a developmental point of view. The aims of the study were: to analyse the influence of empatby and anxiety on cooperative interactions; to explore developmental differences on subjects' cooperative courses of action when the existing relation was of mutual frienship or rejection. Interactions were analysed by means of the 'Lag Sequential Analysis' (Sackett, 1980) in order to leam more about subjects' interactive processes. Different behavioural pattems were obtained for children and adolescents on the basis of the empathy and friendship that existed between subjects.
\end{abstract}

Key words: Empathy; Friendship; Cooperation; Competition; Children; Adolescents.

Agradecimientos: Agradecemos al Dr. Félix López sus sugerencias y reflexiones sobre algunos aspectos «peliagudos» de este artículo. Así como las recomendaciones realizadas por la redacción de Infancia y Aprendizaje y dos revisores anónimos.

Correspondencia con autores: Universidad de Málaga. Dpto. de Psicología Evolutiva y de la Educación. Facultad de Psicología. Campus de Teatinos. 29071 Málaga.

Original recibido: abril, 1990. Revisión recibida: diciembre, 1992. Aceptado: noviembre, 1992. 


\section{INTRODUCCION}

Las nuevas corrientes de la Psicología han criticado con dureza y lucidez:

«EI hábito de trazar fuertes límites conceptuales entre el pensamiento, la acción y la emoción como "regiones" de la mente, y tener luego que construir puentes conceptuales para conectar lo que nunca se debería haber separado» (Bruner, 1986; p. 112).

Con la pretensión de debilitar ese hábito, nuestro estudio se propone analizar los efectos conjuntos que la empatía, la ansiedad y la amistad imprimen sobre los cursos de acción cooperativos y/o competitivos de los sujetos. La elección del término cursos de acción (Fierro, 1986) implica, de forma tácita, un compromiso con las pretensiones teóricas de lo que ha venido a denominarse Psicología de la Acción, y que ha encontrado su expresión en ámbitos de investigación tan diferentes como la Psicología Social (Shotter, 1983; Harré, Clarke y De Carlo, 1985; Ibáñez, 1985), la Psicología de la Personalidad (Bandura, 1986; Fierro, 1986) y la Psicología Evolutiva (Vygotski, 1934; Bruner, 1986; Scribner, 1986) ${ }^{1}$.

La Psicología de la Acción, en contraposición a la Psicología Cognitiva al uso, no está interesada en la conducta estática del sujeto como producto de unos procesos cognitivos estables, sino en cómo la acción se hace diferente en el curso de sus interacciones con otras acciones, ya sean propias o ajenas.

Este será el enfoque conceptual y metodológico con el que estudiaremos las variables que influyen en las secuencias de conducta cooperativa de las personas.

Ante la ausencia de teorías explicativas específicas que permitan predecir la influencia de los pensamientos y sentimientos de los sujetos sobre sus cursos de acción cooperativos y competitivos, hemos optado por aplicar al estudio de la cooperación el modelo explicativo de la ayuda defendido por Batson y Coke (1981) y Fuentes (1989), dado que es uno de los modelos más desarrollados y analizados en la investigación de las conductas prosociales. La justificación de dicha aplicación se basa en que existen numerosas investigaciones que relacionan la empatía y la ansiedad con la conducta de ayuda; pero, en cambio, todavía no se ha comprobado qué efectos producirían la capacidad empática y los sentimientos de ansiedad sobre las conductas cooperativas.

Estos autores han encontrado que cuando una persona percibe que otra necesita ayuda es probable que se sienta inclicado a ayudarla. Pero dependiendo del tipo de estado motivacional que sienta (empatía o ansiedad) se considerará que la ayuda ha sido, respectivamente, altruista o egoísta. Desde este esquema explicativo la conducta de ayuda estará mediatizada por los sentimientos de empatía y de ansiedad suscitados por el contexto o «las condiciones experimentales» (ver Figura 1).

Pero lo novedoso y primordial de este modelo no radica en proponer que una persona ayudará a otra cuando perciba que se encuentra en una situación de necesidad o desamparo, sino en cuestionarse la razón o razones por las que se ayuda. Tal como se expresa en la Figura 1, en algunas ocasiones la ayuda está producida por la empatía (experiencia vicaria de los sentimientos de otra persona) y en otras por la ansiedad. Cuando es la empatía la que origina la ayuda se dice que ésta es altruista, porque su motivación es disminuir el malestar de la otra persona. En cambio, cuando la ayuda esta provocada por la ansiedad, se considera que la ayuda es egoísta, porque el objetivo de la ayuda consiste en eliminar el propio malestar del observador.

La idea de que la empatía y la ansiedad provocan la conducta de ayuda ha 


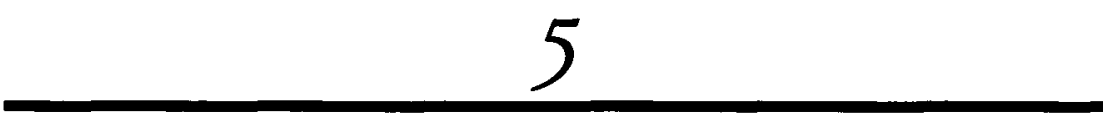

FIGURA 1

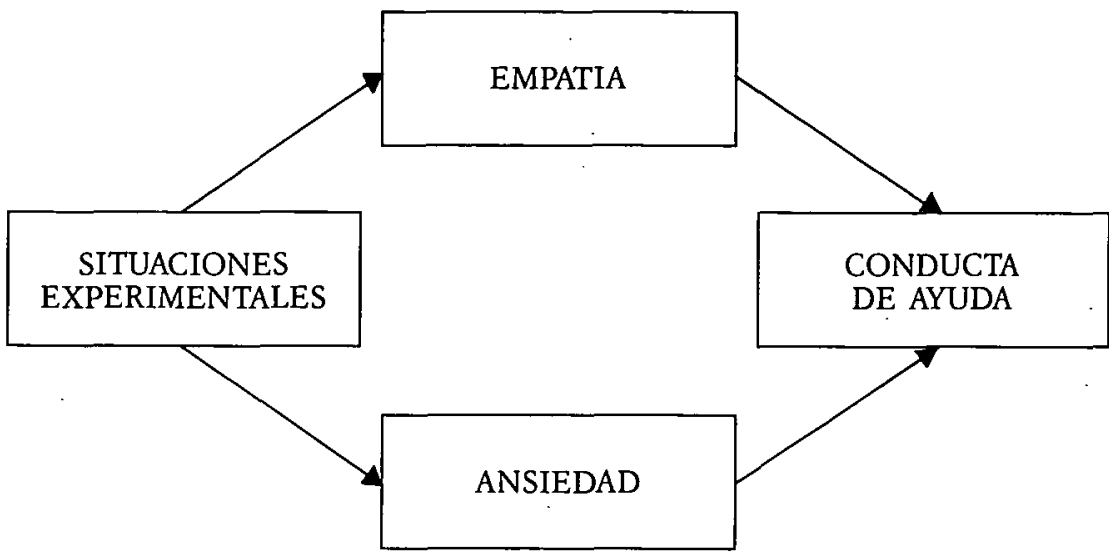

Modelo propuesto por Batson y Coke (1981) para explicar las variables (empatía y ansiedad) que mediatizan la conducta de ayuda

sido defendida por numerosos autores, entre los que podemos destacar a Hoffman (1977, 1982), Eisenberg y Miller (1987) y Batson y Coke (1981), pero ellos únicamente han analizado la influencia de estas variables afectivo-motivacionales en relación al comportamiento prosocial de ayuda. En cambio, nuestro estudio lo que pretende es aplicar este modelo a los comportamientos cooperativos, que buscan el beneficio común, frente a los prosociales, que pretenden el bienestar del otro.

La amistad, tercera variable considerada en nuestro estudio, ha sido tradicionalmente abordada por los investigadores desde el punto de vista de las representaciones mentales que tienen los niños sobre sus relaciones de amistad (Hartup, 1983, 1984; Rubin, 1981; Selman, 1981; Youniss y Wolpe, 1978). Pero existen pocas investigaciones que analicen cómo se comportan las personas realmente con sus amigos y en qué se diferencian esas conductas de las que se mantienen con los no amigos. Ya existen algunos estudios observacionales sobre este tema realizados con niños de preescolar (Marcus, Telleen y Roke, 1979; Iannotti, 1985) y nuestro estudio se propone hacerlo con niños de 10 y 14 años.

Se parte, por tanto, de que los comportamientos prosociales y cooperativos constituyen la base de las relaciones de amistad entre los sujetos, tal como muestran los estudios mencionados sobre las concepciones de la amistad, y lo que se intenta es comprobar esto en las interacciones reales de los sujetos y ver si estas interacciones difieren conductualmente de las que se tienen con los no amigos.

En resumen, nuestras hipótesis enlazan dos enfoques, por un lado, la propuesta de Batson y Coke sobre la influencia de la empatía y la ansiedad en la conducta prosocial, para comprobar si estas variables también afectan a los cursos de acción cooperativos. Y por otro, los estudios de Rubin acerca de las ideas que tienen las personas sobre la amistad, en el sentido de que los amigos cooperan más entre sí que los no amigos, para analizar si estas creencias determinan su conducta.

Respecto a la variable dependiente, la cooperación, vamos a resumir brevemente las dos posturas que describen la evolución del comportamiento cooperativo: por un lado, los estudios de Madsen (1971) y Kagan y Madsen (1971), que 
han hallado que los niños son menos cooperativos a medida que se hacen mayores. Para dichos autores estas diferencias evolutivas están motivadas por la diversidad en los objetivos educativos y de socialización propuestos, tanto desde la familia como por la escuela, para los niños de distintas edades. A los preescolares generalmente se les enseña a cooperar y ayudar a los compañeros, pero a medida que los niños crecen, el énfasis de la socialización se va desviando hacia exigencias de rendimiento académico y hacia el logro de habilidades individuales.

Las críticas que han recibido estos estudios se basan en el tipo de tareas presentadas a los niños, pues en ellas sólo se les permiten dos respuestas: cooperar o competir con los otros. Por ello, es posible que lo que reflejen estos hallazgos en relación a la disminución evolutiva de la cooperación sea simplemente un aumento de la competitividad al crecer los niños, sin que ello sea en detrimento de la cooperación. El niño puede aumentar su competitividad en determinadas situaciones sin disminuir necesariamente su cooperación en otras circunstancias.

Por otro lado, la postura defendida por Piaget (1964) mantiene que la evolución de la cooperación va ligada a determinados avances que se producen a nivel cognitivo, en especial la capacidad de descentración, el desarrollo de la toma de perspectiva social y la coordinación progresiva de un mayor número de puntos de vista, en el sentido de que la cooperación mejoraría cuando se produjeran dichos logros cognitivos.

Algunos autores interesados en el estudio de las amistades infantiles (Youniss y Volpe, 1978; Youniss, 1980) han investigado la evolución de la reciprocidad como un tema crucial para comprender las interacciones entre pares. Su aportación se centra en la evolución de la cooperación, especialmente la diferenciación entre reciprocidad simétrica y preciprocidad cooperativa.

La primera consiste en un intercambio social que implica «dar y recibir» cantidades equivalentes de cosas, que coincidiría con la forma de cooperación designada por Axelrod (1984) como estrategia de «toma y daca» y que se aprecia con frecuencia en el «dilema del prisionero». Youniss y Volpe (1978) piensan que este tipo de intercambio o trueque es de carácter inmediato, no admite la dilación en el tiempo y es propio de los niños comprendidos entre los 6 y los 9 años.

En cambio, la reciprocidad cooperativa comienza a partir de los 9 años y se caracteriza por ser una decisión selectiva en el sentido de que el sujeto decide cooperar con algunas personas y no con otras dependiendo no del tipo de intercambios materiales, sino del tipo de relación que existe entre los sujetos. Además, no requiere la inmediatez en los intercambios, sino que los actos cooperativos se inscriben en un cotexto relacional duradero.

En nuestro estudio analizaremos si los sujetos adoptan estrategias de «toma y daca» en sus interacciones o si optan por una reciprocidad cooperativa, en la que se admiten otros tipos de intercambios mediados por la calidad de la relación existente entre los sujetos (amistad o rechazo).

Las hipótesis de este trabajo prevén que:

a. Los sujetos de la condición cooperativa (que leyeron un texto que fomentaba la empatía y la cooperación) obtendrán puntuaciones más altas en empatía que los sujetos de la condición competitiva (que leyeron un texto sobre el éxito y la competición). 
b. La condición cooperativa logrará crear más comportamiento cooperativo que la condición competitiva.

c. A mayor grado de empatía se producirán más comportamientos cooperativos en los sujetos.

d. Las relaciones de amistad entre los sujetos favorecerán el comportamiento de cooperación, por lo que se espera que los chicos y chicas cooperen más al jugar con sus amigos que al hacerlo con los niños que rechazan. Este pronóstico se hace tanto en relación a los niños de 10 años $\left(4 .^{\circ} \mathrm{EGB}\right)$, como con respecto a los de 14 años (8..$^{\circ} \mathrm{EGB}$ ).

e. Se esperan diferencias evolutivas en comportamiento cooperativo entre los sujetos de 10 y 14 años ( $4 .^{\circ}$ y $8 .^{\circ}$ de EGB respectivamente), en el sentido de que los niños de 14 años muestren más comportamientos cooperativos que los de 10 años.

No se hacen previsiones sobre la variable ansiedad dado que no hay datos para determinar su papel en los cursos de acción cooperativos y competitivos de los sujetos, por lo que respecto a esta variable el estudio es exploratorio.

\section{METODO}

\section{Sujetos}

El estudio se realizó con 170 sujetos de ambos sexos pertenecientes a dos grupos de edad: uno de cuarto curso de EGB (10 años) y otro de octavo curso de EGB (14 años) del Colegio Nacional «José María Torrijos» de Málaga, durante el curso académico 1987-88.

\section{Procedimiento y descripción de las pruebas}

El diseño experimental se realizó en las siguientes fases:

Primero se pasó un sociograma con el fin de conocer quiénes eran amigos y quiénes se rechazaban.

A continuación, dentro de cada grupo de edad, se crearon dos grupos al azar y los sujetos fueron asignados a dos situaciones experimentales, quedando los grupos constituidos de la siguiente forma:

En cuarto de EGB:

a. Situación cooperativa, con 45 sujetos.

b. Situación competitiva, con 43 sujetos.

En octavo de EGB:

a. Situación cooperativa, con 41 sujetos.

b. Situación competitiva, con 41 sujetos.

Cada grupo realizó las siguientes tareas:

A. Lectura y reflexión sobre un texto, diferente según las condiciones experimentales:

a. En la situación cooperativa se leyó un texto que trataba sobre «la unión hace la fuerza» que pretendía fomentar la empatía y la cooperación en los niños.

b. En la situación competitiva los niños leyeron y reflexionaron sobre un texto titulado «Maradona, un triunfador» que trataba del éxito de este jugador.

B. La escala de adjetivos de empatía y de ansiedad, que se pasó a continuación a los dos grupos experimentales, fue el Indice de Empatía y Ansiedad ela- 
borado recientemente por Batson, Fultz y Schoenrade (1987). Elegimos esta prueba porque es la más actual y recoge las aportaciones de los estudios anteriores (Coke et al., 1978; Batson et al ., 1979; Fultz, 1982; Toi y Batson, 1982, y Batson et al., 1983) sobre la diferenciación emocional de la empatía y la ansiedad.

C. Juego de cooperación. Basándonos en los datos del sociograma, se formaron parejas de amigos y de rechazados. Cada pareja jugó (primero con un amigo y después con un niño al que había rechazado) a un juego de cartas que le exigía decidir en cada jugada si quería cooperar o competir con el otro niño.

El juego consistía en que cada sujeto tenía un taco de cartas de color rojo y otro taco de color negro y podía elegir en cada jugada el color que deseaba poner. Poner una carta de color rojo significaba cooperar con el otro y ponerla de color negro significaba competir. Al finalizar el juego, si la última carta colocada era de color rojo el premio se repartía y cada jugador ganaba tres bombones, pero si dicha carta era de color negro sólo ganaba el jugador que la hubiera colocado, que se llevaba todos los bombones.

Los jugadores no sabían cuándo iba a finalizar la partida, porque ello ocurriría independientemente de su conducta, cuando el observador completaba un mínimo de observaciones en su hoja de registro (alrededor de 20 jugadas).

Además de las variables anteriormente estudiadas, nos pareció adecuado añadir dos variables relacionadas con el tipo de pareja que interaccionaba:

a. Se distinguió entre aquellas relaciones en las que los miembros de la pareja se elegían mutuamente como amigos o rechazados en primer lugar, y aquellas relaciones en las que las elecciones eran recíprocas pero en segundo o tercer lugar. En un principio pensamos utilizar sólo las parejas recíprocamente elegidas en primer lugar por los niños, pero al no obtener un número suficiente para realizar el estudio decidimos incluir también las elecciones recíprocas de segundo y tercer orden, aunque controlando si ello influía de alguna manera en las variables dependientes.

b. También se distinguió entre las parejas del mismo sexo (chico-chico o chicachica) y las de distinto sexo (chico-chica), para controlar si este factor influía en el tipo de interacción.

Con cada pareja había un observador que les explicaba las instrucciones del juego y anotaba las jugadas en la plantilla de observación. Cada sujeto realizó una media de 20 jugadas. Si tenemos en cuenta que cada sujeto jugó dos veces, una con un amigo y otra con un rechazado, nos encontramos con que cada sujeto realizó 40 jugadas, aproximadamente, a lo largo del estudio. Si multiplicamos este promedio por el número de sujetos que formaban la muestra nos encontramos con un total de 6.800 jugadas a analizar.

Tal cantidad de datos es necesario reducirla para poder obtener algún tipo de información contrastable y útil de ella. Los recientes avances en la metodología observacional nos permiten emplear poderosas técnicas de reducción de datos tales como el análisis secuencial (Bakeman y Gottman, 1986; Bakeman y Gottman, 1987, y Sackett, 1987); en concreto, para nuestra investigación nos vamos a servir del «Análisis Secuencial de Retardos» (Lag Sequential Analysis) creado por Sackett (1980), del que existe una aplicación como programa estadístico realizada por Quera (NANSEC, 1982) y que se puede encontrar como subprograma en el paquete estadístico MADAP, Micro-Analytic Data Analysis Package, de Kienapple (1986). 
El análisis secuencial de retardos de Sackett, en contraposición a otros métodos secuenciales, presenta dos ventajas principales:

a) se necesita un menor número de datos, y, sobre todo,

b) explora relaciones no absolutas, sino probabilísticas. Flexibilidad que nos permitirá establecer secuencias de interacción que comprendan más de dos eventos en diferentes retardos.

Dicho de otra manera más gráfica, este análisis nos hace saber, por ejemplo, si el sujeto $A$ coopera la probabilidad que hay de que lo haga también $B$; así como calcular las consecuencias de la conducta de $\mathrm{B}$, de nuevo, sobre la conducta de A. Esta probabilidad será hallada para cada uno de los retardos existentes (en nuestro estudio se calcularon dos retardos) y promediada en un valor $\mathrm{Z}$ (denominado usualmente como Zsum), valor que podrá ser tanto positivo como negativo. En caso de que sea positivo, aplicado al ejemplo anterior, significaría que la cooperación del sujeto $\mathrm{A}$ excita la cooperación del sujeto $\mathrm{B}$; y si hubiera sido negativo, que la cooperación de A inbibe la cooperación de B.

La ventaja primordial de los valores Zsum es que permiten como señalan Bakeman y Gottman (1987; pág. 819) que lo observacional pueda ser también experimental. Lo que traducido a nuestro diseño significa que los datos obtenidos para los cursos de acción de cada pareja podrán ser considerados como múltiples variables dependientes.

\section{RESULTADOS}

Los resultados y niveles de significación que se presentan, de forma muy simplificada, son el producto de una regresión múltiple de todas las variables del estudio sobre los valores Zsum de cada uno de los cursos de acción posibles, a excepción de los contrastes intrasujeto realizados para comparar los cursos de acción de un mismo sujeto en virtud de si jugaba con un amigo o un rechazado.

\section{Resultados de los niños de $\mathbf{1 0}$ años en los cursos de acción cooperativos y com- petitivos}

Los resultados obtenidos con respecto al grupo de 10 años nos muestran que la única variable del estudio que influyó 'en los cursos de acción cooperativos y competitivos fue la composición por sexos de las parejas, independientemente de si las parejas estaban formadas por amigos o por sujetos que se rechazaban. En concreto, si las parejas estaban formadas por componentes de diferente sexo mostraban mayor tendencia a ejercitar cualquier tipo de conductas que las parejas formadas por sujetos del mismo sexo. Es decir, cuando los sujetos cooperaron lo hicieron con mayor intensidad cuando su pareja era del otro sexo; pero esto también se volvía a producir cuando los sujetos competían, de forma que competían con más intensidad cuando su oponente era de sexo distinto al suyo. Por tanto, la ocurrencia de cualquier curso de acción cooperativo o competitivo fue más probable en aquellos juegos en los que los sujetos que participaban eran de sexos diferentes (las diferencias fueron significativas para todos los cursos de acción con una probabilidad del .0001).

Con respecto a las divergencias existentes entre los cursos de acción emitidos por los sujetos en virtud de si jugaban con amigos o rechazados no se encontró ninguna diferencia significativa. A excepción de las conductas de 
competición del sujeto $\mathrm{A}$. La probabilidad de que A volviese a competir tras haber competido antes (por tanto, independientemente de lo que el sujeto B hiciese) fue mayor cuando las parejas estaban formadas por sujetos que se rechazaban $(T=1.7187 ; \mathrm{p}<.05)$.

Resultados de los niños de 14 años en los cursos de acción cooperativos y competitivos

Los resultados obtenidos con respecto al grupo de 14 años son más complejos e interesantes, pues podemos observar un mayor número de efectos significativos.

\section{Cuando los que juegan son amigos}

Los datos referentes a los cursos de acción más probables se expresarán en un formato de proposiciones condicionales para facilitar su comprensión. Pondremos como ejemplo los resultados para la variable empatía:

$$
\begin{gathered}
\text { entonces recursión } \\
\text { Si } A \text { coopera } \rightarrow B \text { coopera } \leftrightarrow B \text { coopera }
\end{gathered}
$$

¿Cómo se interpretan? Bien, cuando una acción de uno de los sujetos provoque otra acción, ya sea del otro sujeto o de él mismo, se expresará mediante un condicional. En el ejemplo anterior:

\section{entonces \\ Si A coopera $\rightarrow$ B coopera}

Pero la cadena de acciones puede seguir, es decir, la acción de $\mathrm{B}$, siguiendo con el ejemplo, puede suscitar nuevas acciones. En tal caso se expresarán de la misma forma, mediante un condicional enlazado al anterior.

$$
\text { Si } A \text { coopera } \rightarrow B \text { coopera } \rightarrow B \text { coopera }
$$

En caso de que las acciones de un sujeto sean independientes de lo que haga el otro, lo que se conoce como recursión, se representarán con una flecha biunívoca:

$$
\text { recursión }
$$

$$
\text { Si B coopera } \rightarrow \text { B coopera }
$$

En relación a los restantes resultados, nos encontramos que la empatía suscitó también cursos de acción competitivos:

Si A compite $\rightarrow$ B compite $\rightarrow$ B compite

La condición experimental de cooperación provocó:

$$
\begin{aligned}
& \text { Si A coopera } \rightarrow \text { B coopera } \\
& \text { Si A compite } \rightarrow \text { B compite }
\end{aligned}
$$

La condición experimental de competición excitó los cursos de acción:

$$
\text { Si A coopera } \rightarrow \text { B compite } \rightarrow \text { A compite } \rightarrow \text { B coopera }
$$

La ansiedad incidió en que:

$$
\text { Si B coopera } \rightarrow \text { B coopera }
$$


Con respecto a la relación entre los amigos (si eran recíprocos o no) cabe decir que el hecho de que la relación de amistad no fuera recíproca excitó primordialmente conductas competitivas:

$$
\text { Si A coopera-B compite }-A \text { compite }
$$

La variable sexo nos muestra un efecto curioso. Si B coopera es más probable que la conducta de $A$ sea de cooperación en los hombres que en las mujeres; pero si B en lugar de cooperar compite, la respuesta de competir por parte de A es también más probable en los hombres que en las mujeres.

La composición por sexos de las parejas incidió en los cursos de acción de una forma similar a como lo hizo con los niños de 10 años, pues las parejas mixtas tuvieron tendencia a exagerar sus conductas de cooperación o competición, cuando las hubo, con respecto a los cursos de acción presentados por las parejas del mismo sexo.

\section{Cuando los que juegan se rechazan}

Las variables que afectaron a los cursos de acción de las parejas cuando jugaron con un compañero rechazado se reducen esencialmente a dos: la ansiedad y la composición por sexos de las parejas.

Los sujetos que mostraron una elevada ansiedad tras las condiciones experimentales son los que muestran unos cursos de acción más intensos ( $\mathrm{p}>.05$ ). Dicho de otra forma, los sujetos cuya ansiedad era mayor estuvieron dispuestos cuando A cooperaba a cooperar más que los no ansiosos; pero si A decidía competir, en vez de cooperar, sería también más probable que compitiera que un sujeto con menor ansiedad.

Con respecto a la composición por sexos de las parejas cabe decir que las parejas mixtas tuvieron un efecto parecido al de la ansiedad con respecto a los diferentes cursos de acción (aunque estadísticamente más significativos, $\mathrm{p}>$.0001). Si la pareja de rechazados estaba compuesta por un chico y una chica cualquier patrón conductual se daba con mayor intensidad. Así, si A cooperaba y B cooperaba también, cuando ocurría, se producía con mayor intensidad. Si se trataba de conductas competitivas, ocurría lo mismo.

\section{Diferencias entre los que son amigos y los que se rechazan}

La secuencia $A$ compite $B$ compite es más probable cuando los que forman la pareja se rechazan $(T=-1.9358 ; \mathrm{p}<.05)$, al igual que la secuencia $B$ compite A compite $(T=-1.7294 ; p<.05)$. Es lo que se denomina una secuencia de interacción recíproca, en este caso de competición recíproca. Es esta espiral de mutua competición lo que caracteriza las diferencias existentes entre las parejas de amigos de las parejas de rechazados:

$$
\text { Si A compite }-B \text { compite }
$$

Quizás sea de resaltar un patrón conductual obtenido para los amigos:

$$
\text { Si B coopera } \rightarrow \text { A compite }
$$

Esto es más frecuente en las parejas de amigos que en las de los que se rechazan $(\mathrm{T}=1.9027 ; \mathrm{p}<.05)$, pero sin que genere ningún ciclo de competición recíproca como en los que se rechazan. 
CONCLUSIONES Y DISCUSION DE LOS RESULTADOS OBTENIDOS EN EL ANALISIS MICROANALITICO SOBRE COOPERACION Y COM. PETICION EN NIÑOS DE 10 Y 14 AÑOS

Desde una perspectiva evolutiva, si comparamos los resultados de los niños de 10 años con los obtenidos por los adolescentes, observamos una clara divergencia que consiste en que no se encontraron influencias en el comportamiento competitivo o cooperativo de los sujetos de 10 años en función de las condiciones experimentales ni de los estados motivacionales suscitados por ellas (estados motivacionales que, a su vez, fueron más débiles en los niños de 10 años que en los adolescentes, tal como se refleja también en un estudio de Fuentes, 1989).

Lo único que sistemáticamente influyó en la conducta de los niños de 10 años fue la composición sexual de la pareja (como se explica en los resultados). En cambio, en los niños de 14 años su comportamiento se vio afectado por distintas variables como las condiciones experimentales, la empatía, la ansiedad, el tipo de relación entre los sujetos y el sexo de los miembros de la pareja (se ofrece en la Tabla I un resumen conceptual de las variables que influyeron en los cursos de acción cooperativos y competitivos de los sujetos de 10 años y adolescentes en virtud de las relaciones existentes con sus iguales).

TABLA I

Resumen conceptual de las variables que influyeron en los cursos de acción, cooperativos y competitivos de los sujetos de 10 años y adolescentes, en función de las relaciones existentes con sus iguales

\begin{tabular}{|c|c|c|c|c|c|}
\hline \multicolumn{2}{|c|}{10 AÑS } & \multicolumn{4}{|c|}{ ADOLESCENTES } \\
\hline AMIGOS & RECHAZADOS & \multicolumn{2}{|c|}{ AMIGOS } & \multicolumn{2}{|c|}{ RECHAZADOS } \\
\hline $\begin{array}{l}\text { COOPERACION } \\
\text { COMPETICION }\end{array}$ & $\begin{array}{l}\text { COOPERACION } \\
\text { COMPETICION }\end{array}$ & COOPERACION & COMPETICION & COOPERACION & COMPETICION \\
\hline$\backslash$ & & $\begin{array}{l}\text { Empatía } \\
\text { Cond. Exper. } \\
\text { Cooperativa }\end{array}$ & $\begin{array}{c}\text { Empatía } \\
\text { Cond. Exper. } \\
\text { Competitiva } \\
\\
\text { Relaciones } \\
\text { Amistad no } \\
\text { Recíprocas }\end{array}$ & Ansiedad & Ansiedad \\
\hline Sexo Parejas & Sexo Parejas & $\begin{array}{l}\text { Sexo Parejas } \\
\text { Sexo: Hombres }\end{array}$ & $\begin{array}{l}\text { Sexo Parejas } \\
\text { Sexo: Hombres }\end{array}$ & Sexo Parejas & Sexo Parejas \\
\hline
\end{tabular}

Esto podría ser explicado bajo la hipótesis global de que los niños de 10 años son más dependientes de los aspectos meramente situacionales (como el sexo de la persona con la que interactúan en un momento dado) que los adolescentes, quienes estarían influidos por variables más estables como su concepción de la amistad y los aspectos motivacionales de su conducta.

Desde esta interpretación parece lógico que en los niños de 10 años, y dada la ausencia de factores más estables que guíen su conducta, destaque como un factor dominante en sus interacciones el sexo del compañero. Sobre todo si te- 
nemos en cuenta que durante la infancia existe una clara preferencia por los amigos y compañeros de juegos del mismo sexo. Si durante la infancia las interacciones se dan en grupos unisexuales, podemos pensar que, en nuestro estudio, jugar con un compañero del sexo opuesto tenga un significado distinto para los niños que para los adolescentes, quienes están más acostumbrados a interaccionar con el otro sexo, dada su tendencia a transformar los grupos unisexuales en pandillas heterosexuales.

Maccoby (1985) recogiendo los resultados de numerosos estudios ha resumido del siguiente modo las diferencias en las pautas interactivas entre los grupos de chicos y de chicas durante la infancia: las chicas forman grupos más reducidos (de 2 o 3 personas) que los chicos; en los grupos de chicas es más frecuente la participación de todos los miembros de cara a tomar decisiones o iniciar actividades que en los gupos de chicos, quienes suelen estar más dominados por un líder que decide lo que debe hacer todo el grupo; y, además, las chicas se implican menos que los chicos en juegos de peleas, ataques y luchas, tanto verbales como físicos.

Estas diferencias en los estilos interactivos llevan a Maccoby y Jacklin (1987) a concluir que durante la infancia los niños y niñas viven en «culturas» diferentes dependiendo de su género y apuntan como posibles razones de esta segregación:

- las relaciones de dominio entre los sexos existentes en la sociedad y transmitidas por la familia desde el momento del nacimiento y

- la evitación de las relaciones con el otro sexo por temor a que el grupo las interprete como «románticas» o sospechosas de «intenciones sexuales».

Pensamos que, desde estos argumentos, se explica el hecho de que, en nuestro estudio, el sexo sea el factor más destacado en las interacciones chico-chica a los 10 años y, en cambio, sea un factor más, junto a otros, en la explicación de las interacciones de los sujetos adolescentes.

Respecto a los resultados obtenidos con los adolescentes, aunque no es fácil sacar conclusiones generales a partir de las secuencias conductuales obtenidas en el estudio microanalítico, nos gustaría destacar ciertas pautas observadas en sus interacciones:

a. La empatía sólo tuvo efectos sobre los sujetos que eran am. trando ningún efecto en los cursos de acción de los que se rechazában. De tal forma que, en los sujetos que eran amigos, suscitó secuencias cooperativas siempre que el sujeto A comenzase cooperando (aunque si el sujeto A se atrevía a competir, B le pagaba con la misma moneda).

b. La ansiedad ha revelado que elicitó, al igual que la empatía, efectos diferenciales según el tipo de relación de amistad existente entre los sujètos. De forma que, mientras no jugó ningún papel apreciable en los cursos de acción de los sujetos que eran amigos, las conductas tanto de cooperación como de competición de los que se rechazaban se vieron, en parte, determinadas por los niveles de ansiedad suscitados por las condiciones experimentales.

Como defiende el modelo de Batson y Coke (1981) la empatía provoca ayuda desinteresada, mientras que la ansiedad, cuando provoca ayuda, es de tipo egoísta (es decir, para aliviar la propia ansiedad del sujeto). Nosotros, en un alarde de trasvasar su teoría a otros comportamientos prosociales, hemos intentado generalizar estos presupuestos teóricos a la cooperación. Pero hemos añadido una variable que modula los estados motivacionales de empatía y ansiedad que afec- 
$\tan$ a los comportamientos cooperativos de los sujetos: las relaciones afectivas con los otros (ver Figura 2).

FIGURA 2

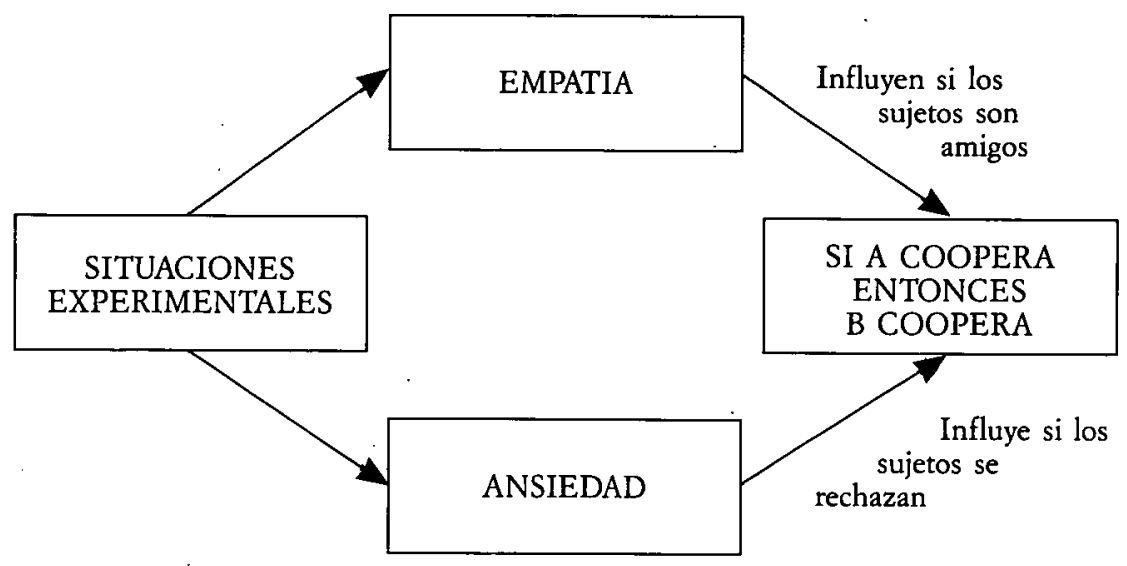

Modelo explicativo del comportamiento de cooperación de los adolescentes, según el cual la cooperación es resultado no sólo de las variables motivacionales (empatía y ansiedad), sino también del tipo de relación afectiva existente entre los sujetos (amistad o rechazo)

Los resultados parecen acordes con el modelo mencionado: lo que nos lleva a actuar de una forma cooperativa o competitiva (además de las acciones del otro) viene dado, por una parte, por lo que sentimos en ese momento y, por otra, por la relación que nos une a la persona que se beneficiará o perjudicará con nuestras acciones.

c. En cuanto a las condiciones experimentales, aparece claramente un patrón conductual para los niños de 14 años que eran amigos: los sujetos que pasaron por la condición competitiva llevaron a cabo secuencias interactivas competitivas; mientras que los sujetos que pasaron por la condición cooperativa estuvieron dispuestos a cooperar siempre y cuando el otro también lo hiciera.

El hecho de que el comportamiento de los adolescentes estuviera modulado por el tipo de relación que existía entre los miembros de la pareja muestra una clara diferencia evolutiva en cuanto a la concepción que tienen los niños y los adolescentes de las relaciones de amistad, y en cuanto a la estabilidad de dichas relaciones. En los niños de 10 años la relación existente entre los sujetos (amistad o rechazo) no influyó de modo significativo en sus interacciones, aunque sí en los adolescentes. Quizás porque en la adolescencia cambia la forma de entender la amistad, pues ésta pasa a ser más íntima, duradera y tiende a estar basada más en la mutua confianza y afecto que en el mero intercambio material o realización de actividades comunes, como ocurre en la infancia. Este nuevo cariz que toman las relaciones de amistad en la adolescencia puede explicar su mayor influencia en las interacciones de cooperación o competición de estos sujetos, frente a su escaso peso en las interacciones de los niños de 10 años (la cooperación pasaría de ser simétrica a recíproca, como diría Youniss, 1980).

En relación a la hipótesis que preveía diferencias entre las parejas de amigos y rechazados con respecto a la cooperación, podemos indicar que se dieron diferencias evolutivas entre los niños de 10 años y los adolescentes como se pre- 
veía. En los niños de 10 años solamente se obtuvieron diferencias entre amigos y rechazados en la competición del sujeto $A$. La probabilidad de que $A$ volviese a competir tras haber competido fue mayor en las parejas de rechazados que en las de amigos. En los adolescentes la mutua competición fue más probable cuando las parejas eran de rechazados que cuando eran de amigos: la competición de A excitó la competición de $\mathrm{B}$, la cual, a su vez, volvió a provocar la competición de A.

Respecto a la hipótesis de que los adolescentes cooperarían más que los niños de 10 años, podemos señalar que los resultados verificaron dicha previsión. Lo cual es congruente con lo defendido por las teorías neopiagetianas sobre la cooperación, aunque las razones apuntadas por Piaget (que los niños a medida que se hacen mayores tienen más capacidad para descentrarse y comprender el punto de vista del otro y, como consecuencia de ello, pueden ser más cooperativos) son diferentes de las razones analizadas y esgrimidas en este estudio. La postura piagetiana relaciona el desarrollo cognitivo con las conductas cooperativas en el sentido de considerar el nivel cognitivo del sujeto como un requisito para poder cooperar; aun aceptando que fuera una condición necesaria, no implicaría que fuese una condición suficiente.

Por ello es posible (como nos han señalado los revisores de este artículo) que variables cognitivas expliquen algunos resultados de este estudio, como, por ejemplo, el hecho de que los adolescentes fueran más sensibles a los efectos de las condiciones experimentales que los niños de 10 años por tratarse de una metodología basada en la presentación de textos.

Nosotros, aun reconociendo que esta u otras variables cognitivas puedan haber influido en el comportamiento de los sujetos de nuestro estudio, nos hemos centrado en otro tipo de variables para explicar la cooperación, variables motivacionales (empatía y ansiedad) y socioafectivas (tipo de relación de amistad o rechazo existentes entre los sujetos), que han mostrado su relevancia en las interacciones de los sujetos. Desde estos resultados, podemos sugerir una integración de factores tanto cognitivos (señalados por la teoría de Piaget) como motivacionales y afectivos (hallados en este estudio) para explicar las diferencias evolutivas entre la infancia y la adolescencia en los cursos de acción cooperativos de los sujetos. Pues, como señalan los psicólogos de la acción, la gente actúa dentro de las limitaciones de lo que «persiensa»: percibe, siente y piensa a la vez (Bruner, 1986).

Esperamos que la posible complejidad de resultados que el lector haya podido encontrar en este estudio microanalítico sea disculpada en aras de la obvia ventaja que supone poder captar con toda precisión lo que ocurre a lo largo de los cursos interactivos. El haber incluido una simple medida de cooperación o competición global hubiera encubierto los procesos y evolución de las acciones de los individuos determinadas por las acciones del «otro», por una parte, y el efecto modulador, por otra, de las variables del estudio.

\section{Notas}

${ }^{1}$ El lector que desee una acepción menos comprometicla, aunque cercana, puede encontrar en Bakeman y Gottman (1986) el témino de secuencias de conuiticta. 


\section{Referencias}

Axelrod, R. (1984). The evolution of cooperation. Nueva York: Basic Books (Trad. cast. Alianza, 1986).

Bakeman, R., y Gottman, J. M. (1986). Observing interaction. An introduction to sequential analysis. Cambridge: Cambridge University Press (Trad. cast. Morata, 1989).

Bakeman, R, y Gottman, J. M. (1987). Applying observational methods: Asystematic view. En J. Osofsky (Ed.). Handbook of infant development. Nueva York: Wiley.

Bandura, A. (1986). Social foundations of thought and action. Englewood Cliffs, NJ: Prentice-Hall (Trad. cast. Martínez Roca, 1987).

Batson, C. D., y Coke, J. S. (1981). Empathy: a source of altruistic motivation for helping? En J. P. Rushton y R. M. Sorrentino (Eds.). Altruism and belping bebavior: social, personality and developmental perspectives. Nueva Jersey: LEA.

Batson, C. D.; Fultz, J., y Schoenrade, P. A. (1987). Distress and empathy: two qualitatively distinct vicarious emotions with different motivational consequences. Joumal of Pessonality, 55 , 19-39.

Batson, C. D.; Harris, A. C.; McCaul, K. D.; Davis, M., y Schmidt, T. (1979). Compassion or compliance: Alternative dispositional attributiones for one's helping behavior. Social Psychology Quarterly, 42, 405-409.

Batson, C. D.; O'Quin, K. F.; J. VAnderplas, M., y. IsEn, A. M. (1983). Influence of self-reported distress and empathy on egoistic versus altruistic motivation to help. Joumal of Personality and Social Psychology, 45, 706.718.

Bruner, J. (1986). Actual minds, possible worlds. Cambridge, Mass.: Harvard University Press. (Trad. cast. Gedisa, 1988).

Coke, J. S.; Batson, C. D., y McDavis, K. (1978). Empathic mediation of helpthg: a two-stage model. Journal of Personality and Social Psychology, 36, 752-766.

Einsenberg, N., y Miller, P. A. (1987). The relation of empathy to prosocial and related behaviors. Psycbological Bulletin, 101, 91-119.

Fierro, A. (1986). El estudio del curso de la acción (Mimeo). Málaga: Departamento de Psicología.

Fuentes, M. J. (1989). Análisis evolutivo de la empatía y la ansiedad como variables mediadoras del comportamiento de ayuda. Infancia y Aprendizaje, 48, 65-79.

FuLTz, J. (1982). Influence of potential for self-reward on egoistically and altruistically motivated belping. Tesis de master no publicada. Universidad de Kansas.

Harre, R.; Clarke, D., y De Carlo, N. (1985). Motives and mechanisms. An introduction to the psychology of action. Londres: Methuen (Trad. cast. Paidós, 1989).

Hartup, W. W. (1983). Peer relations. En P. H. Mussen (Ed.). Handbook of child psychology. Vol. 4. Nueva York: John Wiley \& Sons.

Hartup, W. W. (1984). Las amistades infantiles. En M. C. A. Marchesi y J. Palacios (Eds.). Psicología evolutiva. Tomo II. Madrid: Alianza Psicología.

Hoffman, M. L. (1977). Moral internalization: Current theory and research. En L. Berkowitz (Ed.). Advances in Experimental Social Psychology. Vol. 10. Nueva York: Academic Press.

Hoffman, M. L. (1982). The measurement of empathy. En C. E. Izard (Ed.). Measuring emotions in infants and children. Cambridge: Cambridge University Press.

IAnNorti, R. J. (1985). Assessments of prosocial behavior in preschool children. Developmental Psychology, 21, 46-55.

IBÁÑE, T. (1985). New look en psicología social: Teoria de la acción y autoorganización. Barctlona: UAB.

Kagan, S., y Madsen, M. C. (1971). Cooperation and competition of Mexican, Mexican-American, and Anglo-American children of two ages under four instructional sets. Developmental Psycho$\log y, 5,32-39$.

KIenapple, K. (1986). MADAD. Micro-Analytic Data Analysis Package. Mount Saint Vincent: University Canada.

MACCOBY, E. E. (1985). Social groupings in childhood: their relationship to prosocial and antisocial behavior in boys and girls. En D. Olweus, J. Block y M. yadke-Yarrow (Eds.). Development of antisocial and prosocial bebavior: Theories, research and issues. San Diego: Academic Press.

MaccobY, E. E., y JACKLIN, C. N. (1987). Gender segregation in childhood. En H. W. Reese (Ed.). Advances in child Development and Behavior. Vol. 20. Nueva York: Academic Press.

Madsen, M. C. (1971). Developmental and cross-cultural differences in the cooperative and competitive behavior of young children. Joumal of Cross-Cultural Psychology, 2, 365-371.

Marcus, R. F.; Telleen, S., y Roke, E. J. (1979). Relation between cooperation and empathy in young children. Developmental Psychology, 15, 346-347.

Plaget, J. (1964). Six études de psychologie. París: Gonthier (Trad. cast. Barral, 1971).

Quera, V. (1982). NANSEC. Dept. de Psicología: Universidad de Barcelona.

Rubin, Z. (1981). Las amistades infantiles. Madrid: Morata.

SACKETT, G. P. (1987). Analysis of sequential social interaction data: Some issues, recent developments and a causal inference model. En J. Osofsky (Ed.). Handbook of infant develcpment. Nueva York: Wiley. 
SACKETT, G. P. (1980). Lag sequential analysis as data reduction tecnique in social interaction research. En R. K., L. Walker, D. Sawin y J. Penticuff (Ed.). Exceptional infant. Vol. 4. Nueva York: Brunner-Mazel.

SCRiBner, S. (1986). Thinking in action: some characteristic of practical thought. En R. Sternberg y R. Wagner (Eds.). Practical intelligence: Nature and origins of competence in the everyday world. Nueva York: Cambridge University Press.

Selman, R. L. (1981). The child as a friendship philosopher. En S. R. Asher y J. M. Gottman (Ed.). The development of children's frienships. Cambridge: University Press.

SHOTTER, J. (1983). «Duality of structure» and «intentionality» in an ecological Psychology. Journal for the Theory of Social Bebavior, 13, 19-43.

ToI, M., y Batson, C. D. (1982). More evidence that empathy is a source of altruistic motivation. Joumal of Personality and Social Psychology, 43, 281-292.

VyGoTski, L. S. (1934). Thought and language. Cambridge: MIT Press (Trad. cast. en Pléyade, 1973).

Youniss, J. (1980). Parents and peers in social development: a Sullivan-Piaget perspective. Chicago: University of Chicago Press.

Youniss, J., y VolpE, J. (1978). A relational analysis of children's friendships. En W. Damon (Ed.). New direction for child development. Londres: Jossey-Bass Publisher.

\section{Extended Summary}

The aim of this work was the developmental analysis of students' cooperative or competitive courses of action. Affective variables such as empathy, anxiety, and existing friendships or rejection between 10-14 year old subjects were controlled. These variables were chosen for two reasons:

a) To find out if Baston and Coke's (1981) proposals on the influence of empathy and anxiety in prosocial helping behaviour were also fulfilled with other type of prosocial behaviour such as cooperation.

b) To study in what way existing relations between subjects (mutual friendship or rejection) affect their play interactions.

Children and adolescents' interactions were analysed by means of the 'Lag Sequential Analysis' (Sackett, 1980) in order to study the dynamics of the processes taking place during interaction.

In each age group, subjects were randomly assigned to either of two possible experimental conditions. In one condition, empathy was fostered through reading and analysing a specific text; in the other condition, the same text was read and treated objectively. Furthermore, pairs of friendship and rejection relations between subjects were formed on the basis of the results of a sociogram. Each member of a pair played (first with a friend and afterwards with a child he/she had rejected) a card game requiring the subject to decide at each move whether he/she wanted to cooperate or compete with the other child.

Different behaviour patterns were obtained for both the children and adolescents on the basis of: the experimental conditions to which they had been assigned, and on the type of existing relation (friendship or rejection) between them.

The most outstanding differences between courses of action taken by subject pairs who were friends or who rejected each other were that when there was rejection the following sequence of mutual competion occurred: Sequence, A competes, B competes; and, sequence, B competes, A competes. This spiral of mutual competition did not occur when the subject pairs were friends.

Comparisons between the results obtained for the children with those obtained for the adolescents produce a clear developmental divergence: No behavioural influences were found in the 10 year old children on the basis of the experimental condition. In other words, there were no significant differences 


\section{8}

in the behaviour of the children that were assigned to either the cooperative condition or the competitive condition. The only factor that systematically influenced the behaviour of the 10 year olds was the sex of subject pairs. The behaviour of the 14 year old adolescents however was affected by different variables such as: The experimental condition, empathy, anxiety, type of existing relation between subjects, and the sex of the members of the pair. 